\title{
An Upper Limit for Birefringence in the Boundary Layer of a Heat Conducting Gas
}

\author{
H. van Houten*, W. A. von Marinelli*, and J. J. M. Beenakker \\ Huygens Laboratory, University of Leiden, The Netherlands
}

Z. Naturforsch. 40 a, 164-168 (1985); received November 14, 1984

The Waldmann-Vestner theory for boundary layer effects in polyatomic gases in the nearhydrodynamic regime predicts that in a rarefield heat conducting gas the boundary layers will be birefringent due to the presence of second rank angular momentum polarization. An experiment has been set up in order to measure the birefringence as a function of the distance to the boundary. However, no effect could be detected for $\mathrm{N}_{2}$ and $\mathrm{CO}_{2}$ within the sensitivity of the experiment. This corresponds to an upper limit for the birefringence, which is a factor 6 smaller than expected. These results are in contrast to the qualitative data reported earlier in the literature; no conclusions can be drawn, however, about the validity of the Waldmann-Vestner theory.

\section{Introduction}

During the last decade there has been much experimental and theoretical interest in boundary layer phenomena in gases consisting of rotating molecules. Such phenomena are, for example, field effects on cross phenomena in the Burnett (or nearhydrodynamic) regime such as the viscomagnetic heat flux [1], the viscomagnetic diffusion flux [2], the thermomagnetic pressure difference [3] and the thermomagnetic torque [4], and finally Knudsen corrections on field effects on transport phenomena in gases [5]. A phenomenological theory for these effects has been developed by Waldmann and Vestner [6-12]. In this theory the differential equations which describe the bulk of the gas (up to Burnett terms) are supplemented with phenomenological boundary conditions valid at the wall. This theory has been reasonably successfull in describing the phenomena listed above. A problem is, however, that the theory inherently contains a number of free adaptable parameters as a consequence of the use of phenomenological boundary conditions. These occur in a complicated way in the theoretical expressions in the cases studied so far. The question therefore arises, whether the agreement obtained is significant.

\footnotetext{
* Present address: Philips Research Laboratories, Eindhoven, The Netherlands.

Reprint requests to Prof. J. J. M. Beenakker, Huygens Laboratory, P.O. Box 9504, 2300 RA Leiden, The Netherlands.
}

Such an impression is supported by the wide range of equally good values found for the parameters (see e.g. $[1,3]$ ), and by the fact that the values found in different experiments seem to be unrelated (compare for example the values found for $\tilde{C}_{\mathrm{a}}$ and $\tilde{C}_{\text {am }}$ obtained from the Knudsen corrections for the viscomagnetic effect [5] with those obtained from the viscomagnetic heat flux experiments [1]).

A more decisive test of the theory can be made by measuring the birefringence which is predicted to occur in the boundary layer of a rarefied heat conducting gas [13]. In this experiment the boundary layer can be studied locally, while the other experiments mentioned are all "gap-averaged" phenomena. Qualitative results of a boundary layer birefringence experiment were published by Oudeman et al. in 1981 [14]. The results did not, however, permit a reliable quantitative analysis.

In this paper new apparatus is described, which was developed in order to investigate the birefringence in the boundary layer of a heat conducting gas as a function of the distance to the wall. In Sect. 2 a short introduction to the theory is given, while the experiment is discussed in Sect. 3.

\section{Theory of Boundary Layer Birefringence in a heat conducting gas}

The theory of boundary layer birefringence (BLBF) has been formulated by Vestner and Beenakker in 1977 [13]. In Fig. 1 a flat plate arrangement for the investigation of this effect is schemati- 

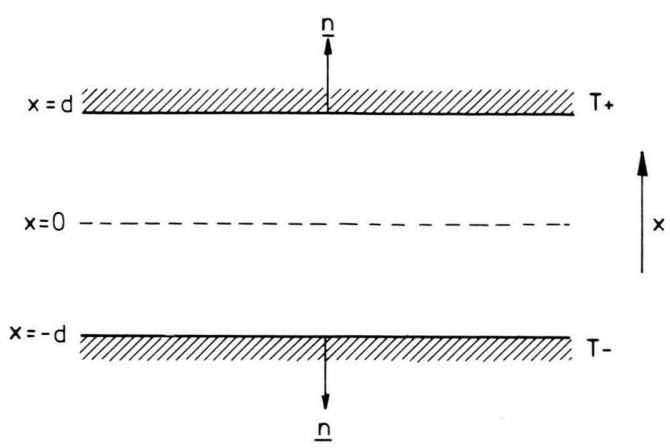

Fig. 1. Schematic parallel plate arrangement with coordinate system for boundary layer birefringence.

cally indicated and a coordinate system is defined. In this theory it is assumed that the dominant angular momentum polarizations occurring in this problem are the tensor polarization $\mathbf{a}=\left\langle\boldsymbol{\Phi}^{02}\right\rangle$, which gives rise to birefringence, and Kagan polarization $\underline{\mathbf{b}}=\left\langle\underline{\boldsymbol{\Phi}}^{12}\right\rangle$, which can be considered as a flux of tensor polarization (the symbol \langle\rangle denotes a non-equilibrium average).

In the bulk of the gas the tensor polarization is zero, since no driving force with the proper tensorial character is present (such as $\nabla \nabla T$, for example). In order to evaluate $\left\langle\boldsymbol{\Phi}^{02}\right\rangle$ in the boundary layer of a heat conducting gas, Vestner and Beenakker [13] derived two differential equations from the transport-relaxation equations which connect the polarizations $\boldsymbol{\Phi}^{02}$ and $\underline{\boldsymbol{\Phi}}^{12}$ according to

$$
\underline{\mathbf{A}}^{12}=-L \boldsymbol{\nabla} \mathbf{A}^{02}+\boldsymbol{q} \cdot \underline{\boldsymbol{\Delta}},
$$

and

$$
\left(1-L^{2} \frac{d^{2}}{d x^{2}}\right) \mathbf{A}^{02}=0 .
$$

The quantities $\mathbf{A}^{02}$ and $\underline{\mathbf{A}}^{12}$, which are proportional to $\left\langle\boldsymbol{\Phi}^{02}\right\rangle$ and $\left\langle\underline{\boldsymbol{\Phi}}^{12}\right\rangle$, are defined in order to give (1) and (2) a simple appearance:

$$
\begin{aligned}
& \mathbf{A}^{02}=p \frac{5}{2}\left\{\frac{k T}{m\left(1+r^{2}\right)}\right\}^{1 / 2} \frac{\{\mathscr{P}(12) \mathscr{P}(02)\}^{1 / 2}}{\mathscr{f}\left(1_{10 \mathrm{E}}^{12}\right)}\left\langle\boldsymbol{\Phi}^{02}\right\rangle, \\
& \underline{\mathbf{A}}^{12}=p \frac{5}{2}\left\{\frac{k T}{m\left(1+r^{2}\right)}\right\}^{1 / 2} \frac{\mathscr{P}(12)}{\mathscr{P}(12 \mathrm{E})}\left\langle\underline{\boldsymbol{\Phi}}^{12}\right\rangle
\end{aligned}
$$

with $r=\left(2 c_{\text {rot }} / 5 k\right)^{1 / 2}$. These definitions are essentially the same as those in [13], although here (as in
[14]) the total heat flux $\boldsymbol{\Phi}^{10 \mathrm{E}}$ has been used as basis function instead of the translational and rotational heat fluxes separately. Hereby simplified expressions are obtained [15]. The quantity $L$ in (1) and (3) denotes a mean free path length which is purely determined by bulk quantities

$$
L=\frac{(\pi)^{1 / 2}}{4 n}\{\mathscr{f}(12) \mathscr{f}(02)\}^{-1 / 2} .
$$

The effective cross sections $\mathscr{f}(02)$ and $\mathscr{f}(12)$ describe the decay of tensor polarization and Kagan polarization, respectively, while the production cross section $\mathscr{A}\left({ }_{10}^{12} \mathrm{E}\right)$ describes the coupling of the total heat flux $\boldsymbol{\Phi}^{10 \mathrm{E}}$ to the Kagan polarization. These cross sections are known from experiments on field effects in the dilute gas regime. Finally the 4 th rank isotropic tensor in (1) has components $\Delta_{i j k l}=$ $\frac{1}{2} \delta_{i k} \delta_{j l}+\frac{1}{2} \delta_{i l} \delta_{j k}-\frac{1}{3} \delta_{i j} \delta_{k l}$.

A non-trivial solution to the set of differential equations (1) and (2) can only be found near the walls, as a result of the boundary condition for the tensor polarization a (or $\mathbf{A}^{02}$ ):

$$
\mathbf{A}^{02}=\tilde{C}_{\mathrm{at}} \boldsymbol{q} \cdot \boldsymbol{n} \overline{\boldsymbol{n} \boldsymbol{n}}+\tilde{C}_{\mathrm{a}} \boldsymbol{n} \cdot \underline{\mathbf{A}}^{12},
$$

which follows from Waldmanns equation for interfacial entropy production. This is the same boundary condition as the one which occurs in the description of the thermomagnetic pressure difference (see [10]). Here $\boldsymbol{n}$ is an outward unit vector normal to the plates (see Figure 1). The symbol- denotes the symmetric traceless part of a tensor. The phenomenological coefficients $\tilde{C}_{\text {at }}$ and $\tilde{C}_{\mathrm{a}}$ are treated as free parameters.

The differential equations (1) and (2) together with the boundary condition (6) yield the following solution (for $L \ll d$ ):

$$
\mathbf{A}^{02}=-\frac{\tilde{C}_{\mathrm{a}}-\tilde{C}_{\mathrm{at}}}{\tilde{C}_{\mathrm{a}}+1} \frac{\sinh (x / L)}{\sinh (d / L)} \tilde{\boldsymbol{n} \boldsymbol{q}} .
$$

The difference $\Delta v$ between refractive indices parallel and perpendicular to $\boldsymbol{n}$ is directly proportional to $\left\langle\boldsymbol{\Phi}^{02}\right\rangle$ (and thus to $\mathbf{A}^{02}$ ), and therefore depends on the position $x$ (see Fig. 1) according to

$$
\begin{aligned}
\Delta v= & \frac{\left(\alpha-\alpha^{\perp}\right)}{10 \varepsilon_{0}} \frac{q}{k T}\left(\frac{m}{3 k T}\right)^{1 / 2}\left(1+r^{2}\right)^{-1 / 2} \\
& \cdot \frac{\mathscr{f}\left({ }_{10 \mathrm{E}}^{12}\right)}{\{\mathscr{f}(12) \mathscr{f}(02)\}^{1 / 2}} \frac{\tilde{C}_{\mathrm{a}}-\tilde{C}_{\mathrm{at}}}{\tilde{C}_{\mathrm{a}}+1} \frac{\sinh (x / L)}{\sinh (d / L)},
\end{aligned}
$$

where $\left(\alpha-\alpha^{\perp}\right)$ is the polarizability anisotropy in S.I. units $\left(F m^{2}\right)$. Near the plates, where $|x| \cong d$, the 
birefringence decreases exponentially with the distance from the wall, i.e. as $\exp -\{(d-|x|) / L\}$.

Local measurements of the birefringence in the boundary layer of a heat conducting gas as a function of the distance to the wall could in principle allow a verification of the theoretical dependence on $x$ as given in (8). Since only bulk properties determine $L$ (see (5)), for this test neither the adaptable parameters $\tilde{C}_{\text {a }}$ and $\tilde{C}_{\text {at }}$ nor the magnitude of the heat flow $q$ need be known.

\section{Experiment}

The measuring cell used to measure BLBF (see Fig. 2) essentially consists of the hot and cold plate arrangement of Fig. 1, mounted in a high vacuum tank. In order to measure the birefringence in the boundary layer locally, use was made of a narrow He-Ne laser beam $(\lambda=632.8 \mathrm{~mm})$. The laser-wall distance could be varied by adjusting the height of the cell with respect to the laser beam, while the optical windows remained fixed. In this way spurious effects due to changes in the optical path were avoided. The optical set-up and detection method are the same as those used by Baas et al. to measure e.g. flow birefringence in gases [16].

The birefringence to be expected is extremely small (predicted value $\Delta v / \Delta T \cong 4 \times 10^{-15} \mathrm{~K}^{-1}$ for

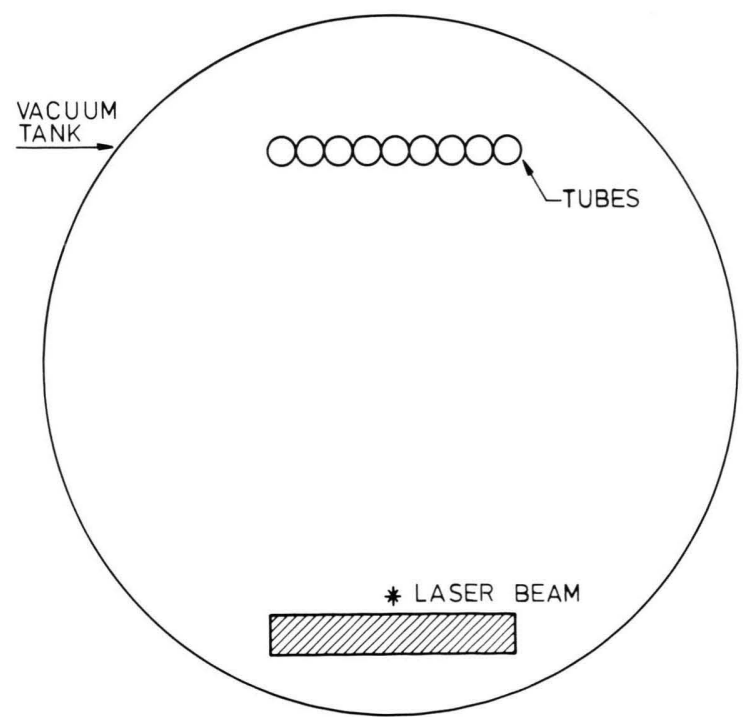

Fig. 2. Cross section of the cell used to measure boundary layer birefringence. As upper plate an assembly of tubes is employed, through which hot and cold water can be circulated for temperature modulation. The laser beam passes perpendicularly to the plane of drawing.
$\mathrm{N}_{2}$ at $1 \mathrm{~Pa}$ and at $2 \mathrm{~mm}$ laser-wall distance for an infinite flat plate arrangement). It is essential, therefore, to modulate the applied temperature difference with a sufficiently short period in order to discriminate against noise and spurious birefringence in the optical elements. A modulation period $<30 \mathrm{~s}$, combined with phase sensitive detection techniques [16], makes it possible in principle to obtain a resolution of $\Delta v \cong 2 \times 10^{-15}$ with the set-up used. In practice the resolution is somewhat worse in this experiment due to disturbances connected with the modulation method used.

The upper plate of the measuring cell consists of a $0.5 \mathrm{~m}$ long assembly of thin walled $(0.2 \mathrm{~mm})$ german silver tubes of $6 \mathrm{~mm}$ diameter (see Figure 2). The temperature modulation was achieved by alternately circulating water from a hot and cold reservoir. With a $30 \mathrm{~s}$ modulation time a $25 \mathrm{~K}$ modulation depth with respect to the lower (glass or brass) plate at $293 \mathrm{~K}$ was easily achieved. Since measurements were only performed near the lower plate, the fact that the upper plate was not flat was not important. The modulation method is not perfect, however, since a phase lag of the temperaturetime profile develops along the plate. This was monitored by a set of Fe-Co thermocouples mounted on the tubes. The effective, phase averaged, modulation depth $\Delta T_{\text {eff }}$ proved to be about $15 \mathrm{~K}$ Since relative measurements of the birefringence as a function of laser-wall distance were intended, a more accurate determination of the modulation depth was not necessary.

Several considerations determine the dimensions of the cell. The birefringence to be measured shows up as a phase difference between two orthogonal components of the incoming linearly polarized laser light. This phase difference is proportional to the cell length, which was taken $0.5 \mathrm{~m}$ in order to obtain sufficient sensitivity. The birefringence was detected near the lower plate. The laser-wall distance had to be greater than $2 \mathrm{~mm}$ in order to avoid problems with scattered laser light. This means that the mean free path $L$ (see (5)) typically had to be between 1 and $10 \mathrm{~mm}$, and that therefore the pressure had to be chosen between 5 and $0.5 \mathrm{~Pa}$ (see (5)). Since furthermore the condition $d \gg L$ should be fulfilled (see Sect. 2), $d=4.5 \mathrm{~cm}$ was chosen, so that the plates were $9 \mathrm{~cm}$ apart. The width of the plates had to be limited to $5 \mathrm{~cm}$, in order to have a reasonably small vacuum tank. Consequently, only a rather 
poor approximation to an infinite flat plate arrangement was obtained, so that the heat flux $q$ was not precisely known. However, this was not essential for the relative measurements intended.

In order to calculate the expected magnitude of the effect a worst case estimate for $q$ can be obtained by comparing the flat plate arrangement of Fig. 2 to a concentric cylinder arrangement; the heated upper plate then corresponds to the inner cylinder, with radius $2.5 \mathrm{~cm}$ and the lower plate combined with the vacuum tank to the outer cylinder with a $11.5 \mathrm{~cm}$ radius. At $2 \mathrm{~mm}$ from the outer cylinder the heat flux $q$ is then found to be a factor 1.8 lower than in the corresponding infinite flat plate arrangement with a $9 \mathrm{~cm}$ gap (see e.g. [17]). This is a rather pessimistic extreme. Moreover, the correction to the heat flux will be smallest at the position of the laser beam near the middle of the lower plate. An upper limit 1.5 for the reduction factor for the heat flux $q$ may therefore safely be assumed.

In the experiments the noise level increased roughly proportional to the temperature modulation depth, and the obtainable resolution was limited to $\Delta v / \Delta T \cong 3 \times 10^{-16} \mathrm{~K}^{-1}$. With $\Delta T_{\text {eff }} \cong 15 \mathrm{~K}$ experiments were performed for $\mathrm{N}_{2}$ and $\mathrm{CO}_{2}$ at various pressures $(0.1$ to $10 \mathrm{~Pa})$ and at various laser-wall distances ( 2 to $9 \mathrm{~mm}$ ). Both glass and brass lower plates were used.

Within the experimental resolution no effect was found. Consequently no information could be obtained about the validity of the differential equation (2). Only upper limits for the combination of $C$-coefficients occurring in (8) have been obtained. Cross section values needed for this purpose were taken from dilute gas experiments. All necessary data may be found in the compilation given in [18]. The Knudsen corrected heat flux $q$ was calculated according to

$$
q=f^{-1}\left(1+\frac{K}{p}\right)^{-1} \lambda \frac{\Delta T}{2 d},
$$

with $K$ the Knudsen correction constant in a flat plate arrangement with a separation of $9 \mathrm{~cm}$ $\left(K=0.4 \mathrm{~Pa}\right.$ for $\left.\mathrm{N}_{2}\right)$ and $f$ the earlier mentioned geometrical reduction factor for $q(f=1.5)$. With the resolution quoted above, the resulting upper limit is (see also (8))

$$
\left|\frac{\tilde{C}_{\mathrm{a}}-\tilde{C}_{\mathrm{at}}}{\tilde{C}_{\mathrm{a}}+1}\right|<0.16,
$$

both for $\mathrm{N}_{2}$ and $\mathrm{CO}_{2}$. This is about a factor 6 lower than expected by using values for $\tilde{C}_{\mathrm{a}}$ and $\tilde{C}_{\text {at }}$ from experiments on the thermomagnetic pressure difference [3]. As discussed in [3] these values are not very reliable, however. A more reliable value for $\tilde{C}_{\mathrm{a}}$ has been obtained from experimentally determined Knudsen corrections on the viscomagnetic effect [5]: $\tilde{C}_{\mathrm{a}}=1.0$ was found, so that from (10) the condition $0.7<\tilde{C}_{\text {at }}<1.3$ can be inferred. No conclusions about the validity of the Waldmann-Vestner theory can be obtained from this result, however.

The upper limit found here is in striking contrast with the large results reported in [14], obtained in a set-up having a resolution (in terms of $\Delta v / q$ ) which was apreciably poorer than in the present experiment. A careful reanalysis of the experimental setup and of the measuring procedure of [14] has led to the conclusion that their results must be attributed to spurious effects.

\section{Acknowledgements}

The stimulating interest of Dr. L. J. F. Hermans is gratefully acknowledged. This work is part of the research program of the Stichting voor Fundamenteel Onderzoek der Materie (Foundation for Fundamental Research on Matter) and was made possible by financial support from the Nederlandse Organisatie voor Zuiver-Wetenschappelijk Onderzoek (Netherlands Organization for the Advancement of Pure Research).

[4] A. L. J. Burgmans and T. W. Adair III, J. Chem. Phys. 59, 324 (1973).

[5] J. N. Breunese, F. W. Gödecke, L. J. F. Hermans, and J. J. M. Beenakker, Physica 126 A, 82 (1984).

[6] L. Waldmann, Z. Naturforsch. 22 a, 1269 (1967).

[7] L. Waldmann, Rarefield Gas Dynamics, Academic Press, New York 1974. 
[8] L. Waldmann, Z. Naturforsch. 32 a, 521 (1977)

[9] H. Vestner, Z. Naturforsch. 28 a, 1554 (1973).

[10] H. Vestner, Z. Naturforsch. 28a, 869 (1973).

[11] H. Vestner, Z. Naturforsch. 29a, 663 (1974).

[12] H. Vestner, Z. Naturforsch. 29a, 365 (1974)

[13] H. Vestner and J. J. M. Beenakker, Z. Naturforsch. 32 a, 521 (1977)

[14] P. Oudeman, J. Korving, H. F. P. Knaap, and J. J. M. Beenakker, Z. Naturforsch. 36a, 579 (1981).
[15] B. J. Thijsse, G. W. 'tHooft, D. A. Coombe, H. F. P. Knaap, and J. J. M. Beenakker, Physica 98A, 397 (1979).

[16] F. Baas, J. N. Breunese, H. F. P. Knaap, and J. J. M. Beenakker, Physica 88A, 1 (1977).

[17] R. P. Feynman, R. B. Leighton, and M. Sands, The Feynman Lectures on Physics, Vol. II, Addison Wesley, London 1964.

[18] H. van Houten, L. J. F. Hermans, and J. J. M. Beenakker, Physica A (1985), to be published. 